\title{
Discovering potent inhibitors against the Mpro of the SARS-CoV-2. A Medicinal Chemistry approach.
}

\author{
Aamir Mehmood ${ }^{1}$, Sadia Nawab ${ }^{1}$, Qiankun Wang ${ }^{1}$, Jiang Xue ${ }^{1}$, Aman Kaushik ${ }^{2}$, and \\ Dong-Qing Wei ${ }^{1}$ \\ ${ }^{1}$ Shanghai Jiao Tong University \\ ${ }^{2}$ Jiangnan University
}

July 29, 2021

\begin{abstract}
The global pandemic caused by a single-stranded RNA (ssRNA) virus known as the severe acute respiratory syndrome coronavirus 2 (SARS-CoV-2) is still at its peak, with new cases being reported daily. Though the vaccinations are done on a massive scale, the frequent mutations in the viral gene and resilience of the future strains could be more problematic. Therefore, there is always a need for new compounds to be available for therapeutic studies. We carried out the present research to discover potential drug compounds against the SARS-CoV-2 main protease. A total of 16,000 drug-like small molecules from the ChemBridge database were virtually screened to obtain the top hits. As a result, 1032 hits were selected based on their docking scores. Next, these structures were prepared for molecular docking, and each small molecule was docked into the active site of the Mpro. Only those compounds with strong interactions with the active site residues and had the highest docking score were subjected to molecular dynamics (MD) simulation. The post-simulation analyses were carried out using the in-built GROMACS commands to gauge the stability, flexibility, and compactness. Principal component analysis (PCA) and hydrogen bonding were also calculated to observe trends and affinity of the drugs towards the target. Among the five top compounds, C1, C3, and C4 revealed strong interaction with the target's active site and remained highly stable throughout the simulation. We believe the predicted compounds in this study could be potential inhibitors in the natural system and must be considered for further practice.
\end{abstract}

\section{Hosted file}

COVID-19 Final draft.docx available at https://authorea.com/users/428064/articles/532063discovering-potent-inhibitors-against-the-mpro-of-the-sars-cov-2-a-medicinal-chemistryapproach 

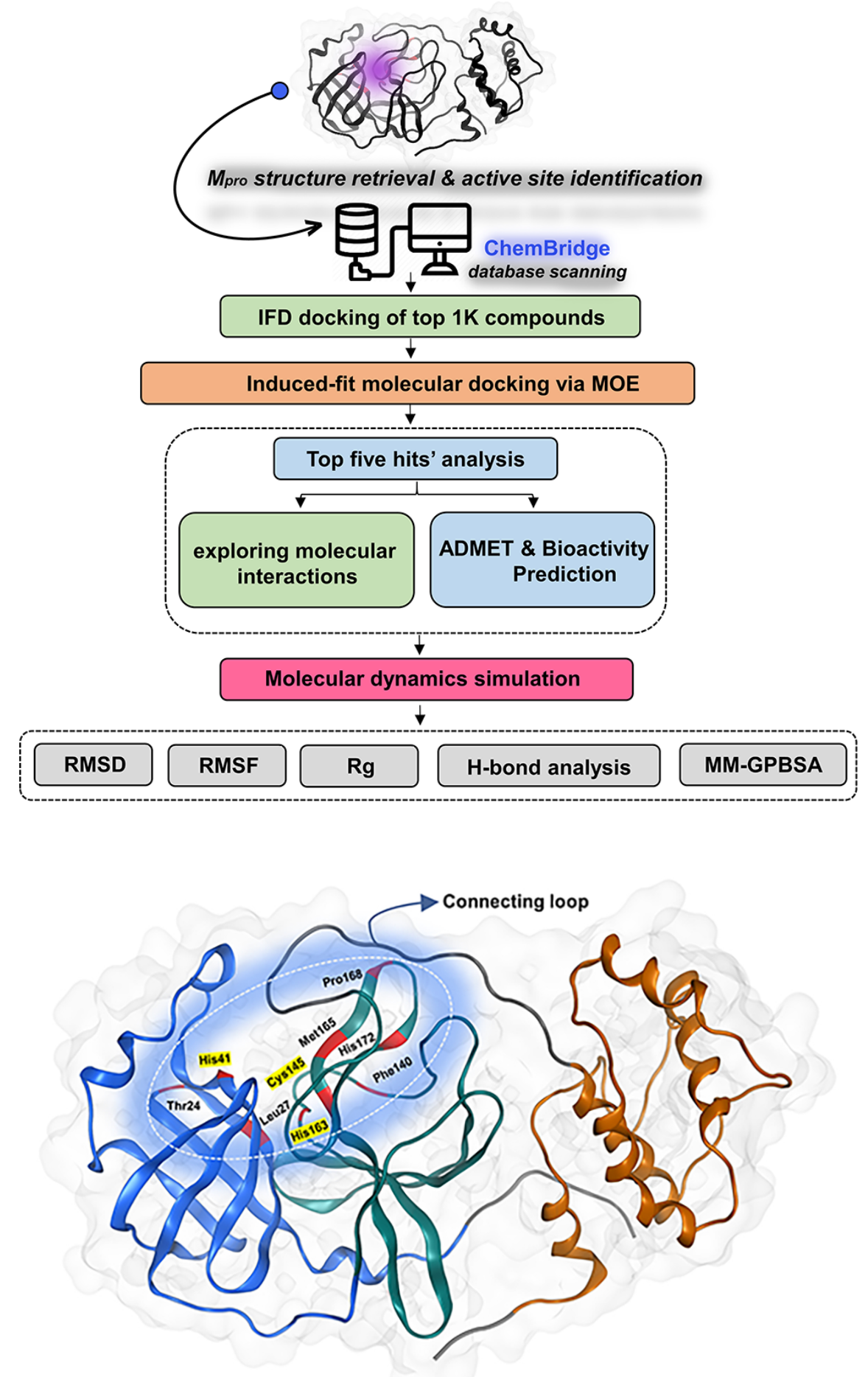

Hosted file

F3 PDF.pdf available at https://authorea.com/users/428064/articles/532063-discovering-potentinhibitors-against-the-mpro-of-the-sars-cov-2-a-medicinal-chemistry-approach 

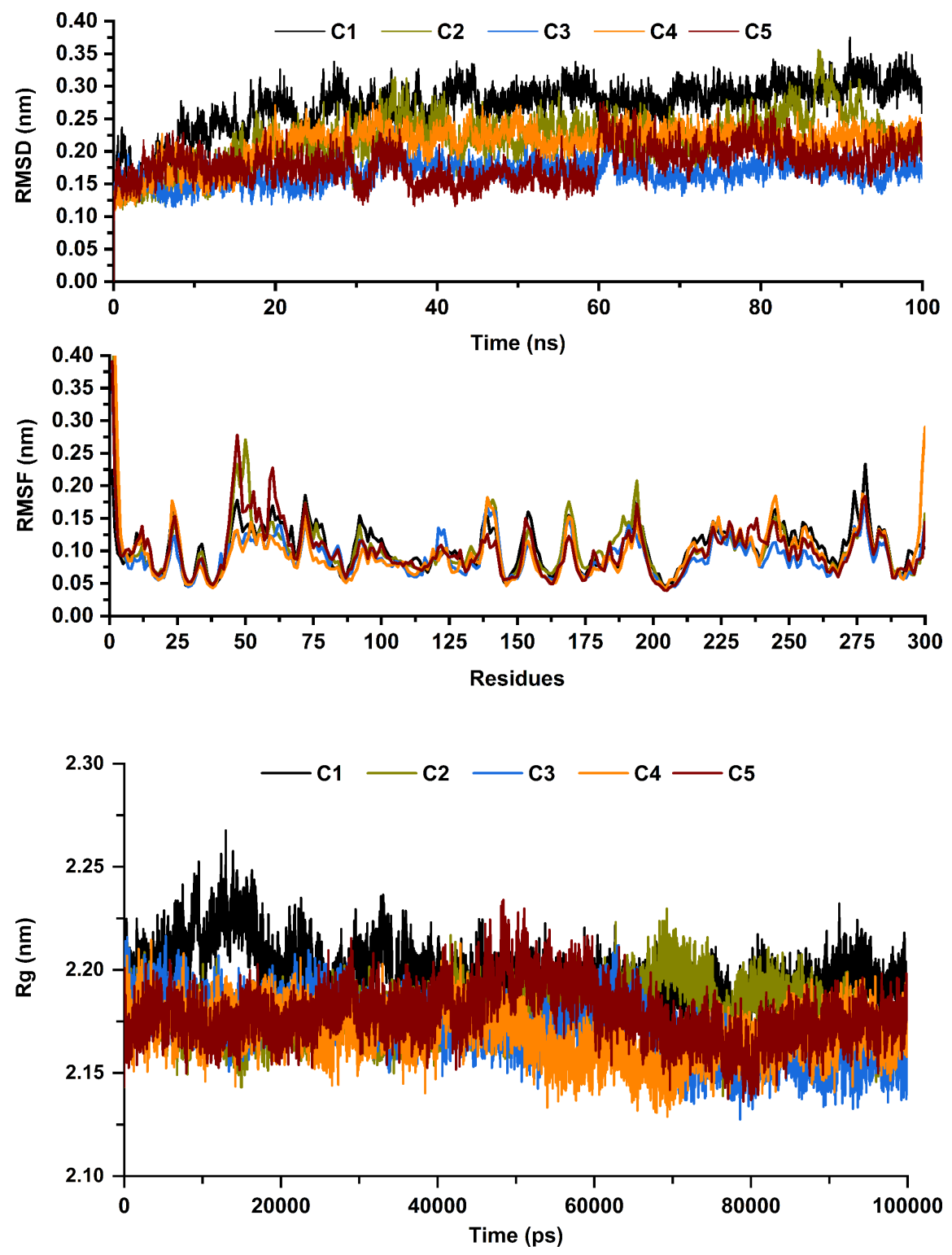

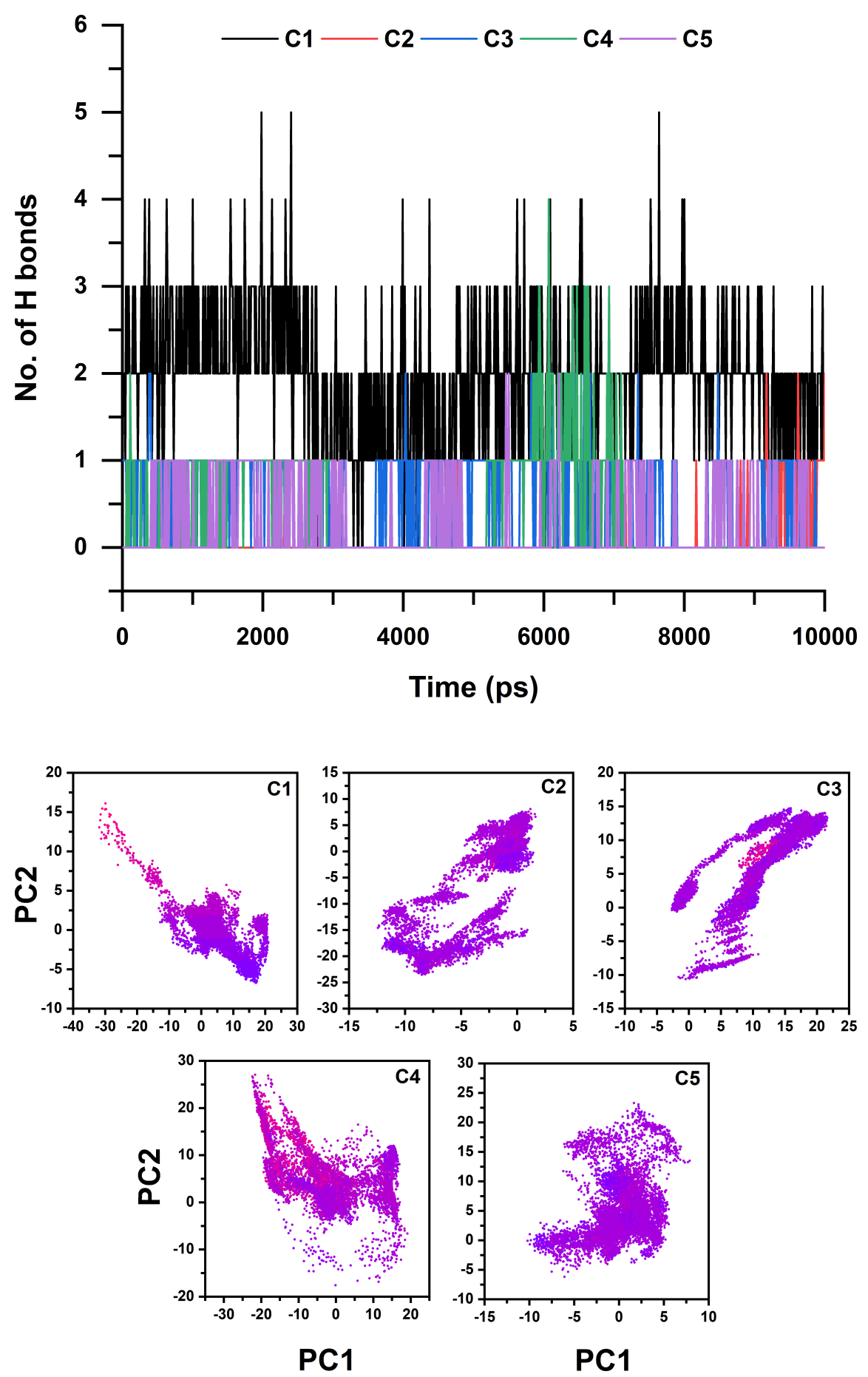

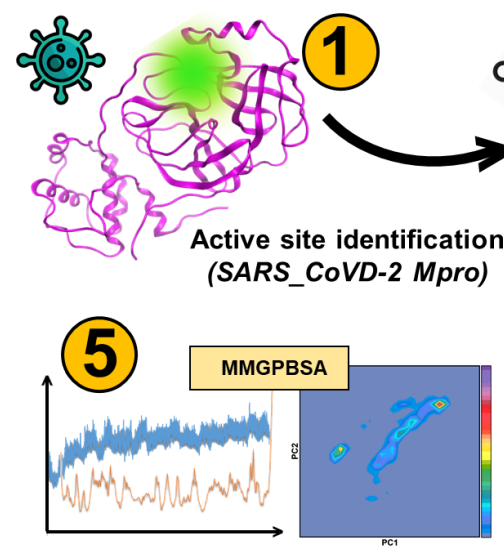

Post-simulation analysis (RMSD, RMSF, PCA, Free energy calculation)
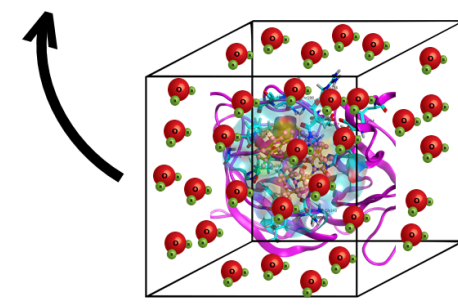

Selection of the top compounds

ChemBridge database

(Virtual screening)
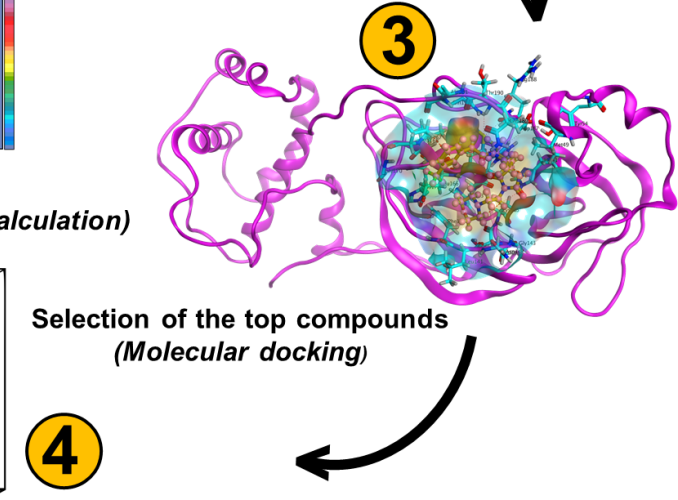
(Molecular docking)

Analyzing stability of the top compounds (Molecular dynamics simulation) 